\title{
TOWARDS SOCIALLY SUSTAINABLE URBAN DESIGN: ANALYSING ACTOR-AREA RELATIONS LINKING MICRO-MORPHOLOGY AND MICRO-DEMOCRACY
}

\author{
RICHARD TIMMERMAN, STEPHEN MARSHALL \& YUERONG ZHANG \\ Bartlett School of Planning, University College London.
}

\begin{abstract}
The social sustainability of cities is increasingly assisted by smart apps, social media and the awareness of how social interactions relate to urban space. Within cities, communities or neighbourhoods are no longer easily spatially defined. Similarly, how a community might govern itself does not necessarily follow traditional, simple, spatially self-contained loci. The role of housing management companies, managing a portfolio of social and private housing, adds additional complexity to relations between individual properties and their collective governance, at a level below that of the local municipality. Meanwhile, the advent of online crowdsourcing and crowdfunding poses new challenges about the influence of outsiders and 'who gets a vote' - and who uses their vote-when making decisions about a neighbourhood's future. This poses a number of challenges for planning and local democracy in the smarter city.

This paper reports on new research from the Incubators of Public Spaces project, involving the use of a novel online design and crowdsourcing platform as an experimental tool for public participation, in the case of a London housing estate. In particular, this chapter analyses relationships between different actors and instruments involved in the governance of the different areas or territories of the housing estate.

We report on the challenges of holistically engaging a focused yet diverse pool of users in the regeneration of a series of courtyards associated with social housing blocks. This involves non-trivial decisions about user access rights within the platform, which becomes a challenge of reinventing a micro-scale democracy. By modifying standard approaches to social network analysis, the paper develops and demonstrates visualisation of the socio-spatial relationships, linking actor networks and area structures, applied in a novel way to a site's micro-morphology. This research, yet in progress, can help inform a new generation of planning procedures for more equitable, inclusive and hence socially sustainable cities.
\end{abstract}

Keywords: Community cohesion, participatory planning, development, management, social network analysis, smart technologies.

\section{INTRODUCTION}

The creation of socially sustainable cities is connected to the involvement of their citizens. According to Haughton and Hunter, 'the principle of participation argues that greater community involvement provides an additional source of knowledge of environmental conditions and improvement needs' [1]. In urban planning this is acknowledged through participatory planning processes. However, traditional approaches, including consultation events/workshops with community representatives, are frequently subjected to criticisms. These include: its inability to attract younger audiences, inconvenient timings and scheduling and failure in being able to substantiate the needs of those directly affected by the processes [2,3]. Therefore, current methodical approaches can be broadly described as exclusionary and detrimental towards facilitating the creation of sustainable urban designs.

The criticisms of participatory planning often relate to social exclusion, where it is described as a tokenistic [3]. At the local scale, notable observations are that communities are only engaged following the conception of a design and that consultations are poorly 
scheduled [4,5]. A by-product of such scenarios is the misrepresentation of involved actors, their areas of influence and potentially erratic applications of policy instruments involved in the dissemination of urban designs. However, the argument is connected to the limited receptivity of informal changes to urban spaces implemented by members of affected communities. The increasing ubiquity of smart technologies coincides with an emergence of research exploring relationships between social interactions and urban space. The challenge is to assess whether smart technologies can be exploited to promote the growth of sustainable urban designs.

The growth of social and smart media opens new opportunities for enhanced spatial modelling that exploits Big Data. Examples are observed in Smart London initiative [6], and the wealth of downloadable consumer apps that provide live updates on transportation networks [7]. However, at present, the application of such data is limited in local-scaled planning, where it could potentially report on:

- The social networks most likely to be impacted by a development, advising on who is the best to target in participatory planning exercises.

- The conditions that lead to marginalisation in community consultations.

- The connections between members of communities and sectors of local and central government and their policies, leading to a better understanding of policy impact.

Spatially, communities are not easily defined or self-contained when depending on conventional planning approaches that rely on interval datasets (census, council registers). In a residential context, housing management companies add a further level of complexity because of their varying associations with local authorities through to individual tenants.

Recently, crowdsourcing and crowdfunding platforms have engaged with smart technologies and have been employed by the commercial sectors as a means of discovering solutions which traditional top-down strategies would have been less likely or even unable to invent [8].

One such approach is that of the Incubators of Public Spaces project (https://www.incubators-of-public-spaces.com) which uses three-dimensional modelling and design software to link to an online crowdsourcing platform which allows public users to have a hand in the redesign of their local neighbourhood.

One of the crucial issues raised by such a system is how it relates to people and planning: it is not just 'design software' wherein an individual could design their own house, lay out their kitchen or garden, but involves the collective creation or reorganisation of public streets and spaces as well as individual buildings. In terms of people, this means there are questions about who gets to use the system and who gets to create and/or vote on design proposals. In terms of planning, it raises questions about how this relates to wider issues of governance, participation and democracy in relation to local plan-making.

If this presents a practical challenge, there is also a more detailed technical or academic challenge, relating to how best to represent and analyse what is going on. Typically, planning processes are presented in terms of flowcharts and tabulations, while people and institutions tend to be represented and analysed as social networks. These are not necessarily directly related to each other or to the geographical territories to which they refer.

Yet, one possible existing approach that combines these is found within the fourth deliverable of the Transport Planning Land Use and Sustainability (TRANSPLUS) project which ran between the years 2000 and 2003 [9]. The purpose of the TRANSPLUS framework was 
to visualise the relationships between the layers of actors, instruments and area typologies across contexts, in a comparative study of transport and land use planning policies in European cities and city regions [9].

This paper explores the application of conceptual TRANSPLUS modelling techniques, to the visualisation of actor, area and instrument relationships at the Pollards Hill Estate, Merton, as part of the analysis of the Incubators project.

\section{THE INCUBATORS PROJECT}

Incubators of Public Spaces ('Incubators'), is an online software platform that provides members of a community a tool to easily review and visualise the layout and design of public streets and places (Fig. 1). The project is a collaboration between KU Leuven (Belgium), Politecnico de Torino (Italy), University College London (UK), ISN and Neurovation (Austria), which seeks to explore ways of engaging broader demographics in participatory planning processes by exploiting smart technologies and living lab methodologies [10]. The premise of the platform is the crowdsourcing of ideas, where users are given opportunities to submit their ideas and have them voted on by the online community of registers. The system is set up so that ideas can be regulated by moderators who essentially enable the regeneration; they have the power to set budgetary limits and define valid intervention typologies.

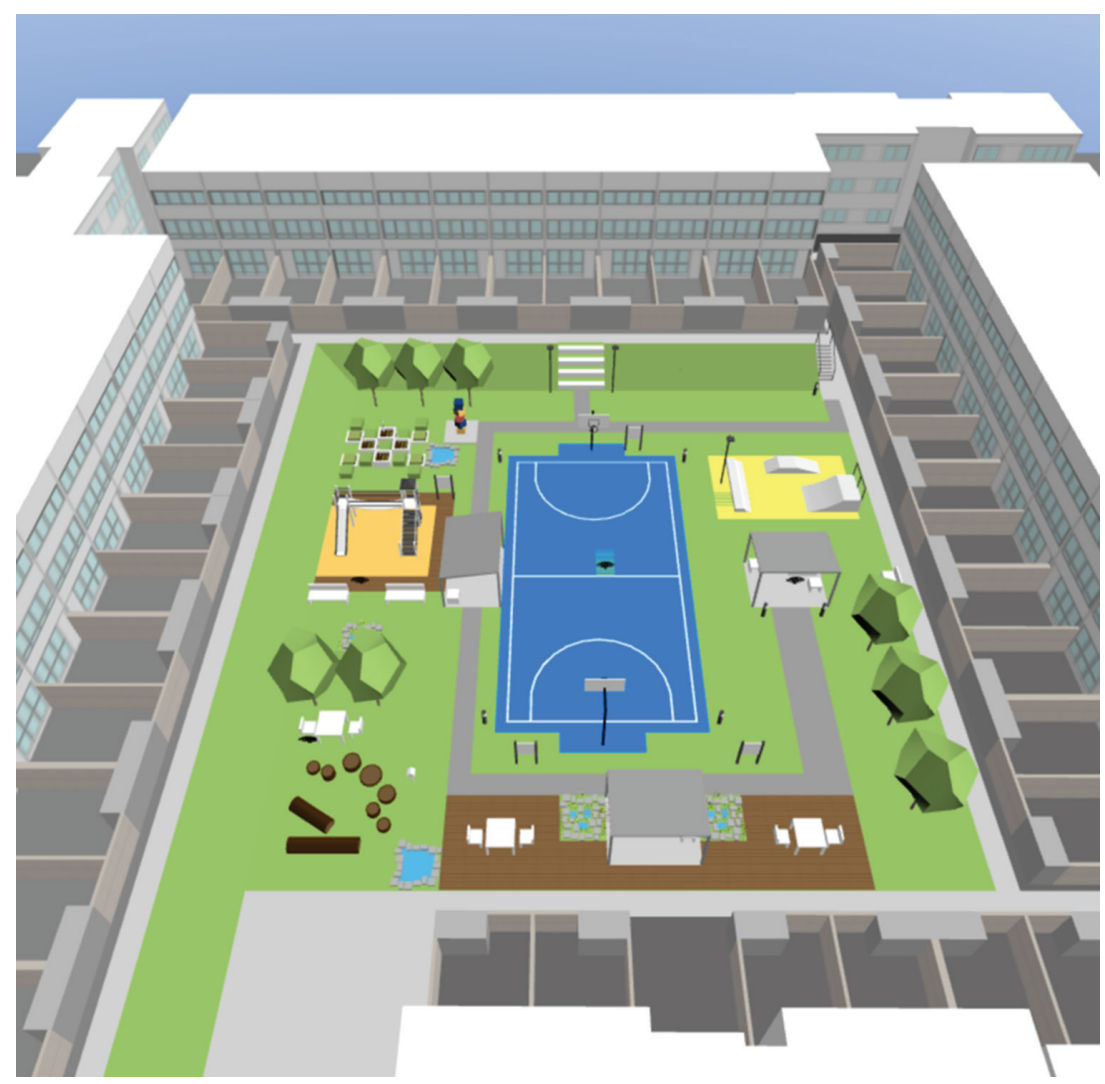

Figure 1: A resident's vision for a courtyard created using the 3D editor. 


\subsection{The Incubators Platform}

The Incubators Platform incorporates crowdsourcing functionalities. Presently, it is designed to run alongside traditional participatory planning processes.

The ergonomics of the platform attempt to simplify web navigation by using clear visual cues. Upon reaching the landing page, first-time users are asked to register during which basic demographic data is collected. After registering, they are able to login and access a home page that briefly describes an applicable regeneration scheme and offers the user an opportunity to submit an idea. A link to an optional questionnaire is included on the landing page to collect further demographic data and feedback towards future enhancements to the system.

Ideas are primarily submitted through an embedded 3D Editor. The 3D editor, a modified iteration of Google Sketch-Up, contains a set of fixed selectable typological interventions that the user can place and manipulate within the editor. Once they have finished their design, they are able to submit their idea to an 'idea pool' visible to other users who have an opportunity to vote or comment on the design. The design achieving the most votes is submitted to the landscape architect in advance of preparing a planning proposal.

\subsection{Pollards Hill}

Incubators have been piloted in three major cities in Europe: Turin, Brussels and London. The London case study is Pollards Hill-a 1970s Housing Estate [9]. This site was selected partly because it has some commonalities and contrasts with the Turin and Brussels cases; additionally, the Incubators project fell conveniently within live consultation phases of its regeneration.

Pollards Hill is an outer suburban housing estate located in the south west London borough of Merton. The case study is ethnically diverse with the majority of its residents possessing an education level of no higher than A-level/GCE [14].

There are nine major courtyards that fall within the scope of the regeneration plans proposed by the presiding Housing Association, Moat. These courtyards are considered under-used/dysfunctional, where interviews reveal Moat's plans to regenerate these as hubs to achieve a sense of community ownership. Moat's consultation process employs traditional participatory planning approaches which involve consultation events and a 'consultation house'; to publicise the events, they incentivised consultations and themed the series 'LOVE Pollards' [11]. However, the response rate to this approach is low, with minimal input from the few community members who attended. Consequently, the proposed landscaping schemes are described as distant from what the community wants.

Regeneration to date includes voting on colour schemes and the recladding of building façades. However, throughout the case study, informal grassroots interventions are apparent such as allotments and the reuse of void spaces into small gardens. These occurrences indicate that consultations aren't necessarily fully representative of residents' needs, for one or more of the following reasons:

- Consultations may not occur at inappropriate times.

- Proposed interventions may fall outside the scope of interest of the community.

- Consultation events may not always reflect a broad spectrum of feedback from the communities affected. 
All of the above could be attributed to the lack of communication, or ineffective consultation drives that could potentially be mitigated through better understanding the relationship between the actors, areas and both formal and informal policies.

One possible approach is conducting social network analysis (SNA). SNA is a popular approach towards visualising the complexity of the relationships between actors, areas and instruments. However, relationships can be visually convoluted and do not necessarily reflect the significance of weak ties that may be most effective apparent changes in the courtyard settings.

Furthermore, there is no representation of the significance of impactful roles played by poorly connected actors such as the silent participants in the shaping of areas and instruments.

\section{BREAKING DOWN THE DIFFERENCES: AREAS, INSTRUMENTS, ACTORS}

The TRANSPLUS framework identifies three distinct elements that can be used to visualise the complexity of the relationships found in its case studies. These elements are: areas, instruments and actors [9].

According to the TRANSPLUS framework, areas are defined as geographic regions that may be of any size. The geographic scales ranged from region through to local authority levels. The framework can also be applied to Incubators although the geographies essentially range from local authority to community courtyards.

Actors are defined as those '(bodies of) people whose members or representatives could in principle be named. They may hold responsibilities or otherwise participate in transport and land use planning processes. Their constituent "unit" is people' [9]. In Incubators, actors include individuals (non-institutional actors) who have an opportunity to participate in the design/development of plans directly affecting them, where the main challenge becomes variety reduction [12]. In a situation of complete autonomy, where Pollards Hill is regarded as a self-contained/self-sufficient community, DIY urbanism is apparent [13]. This results in the identification of 'the silent participant' (introvert), a user who does not interact with the participatory planning tools.

Within the context of TRANSPLUS, Instruments are the active agents that link the areas and actors together. In practical terms, they are the policies and directives that apply at one or more geographic scale. In a similar way to TRANSPLUS, Incubators applies Policy Instruments within a competency framework so that instruments can span a geographic context and act as the sole binding agent between areas and actors-termed as 'authorities' in TRANSPLUS [9].

TRANSPLUS offers an alternative modelling approach (see Fig. 2). It relies on a hybrid networks approach to represent the relationship between actors that is appropriate for clearly showing the relationship between a limited set of actors. However, the adopted approach shows mono-directional relationships between a limited set of actors with a symbology and notations optimised to show relationships between governance/land use and transport.

A key point to take from this kind of diagram is that the structural relationships between areas (e.g. nested ovals) is different from the structural relationships between actors. For example, while the geographical area of Nantes lies within the departmental area of LoireAtlantique, Nantes commune is not a subset of Département Loire-Atlantique department.

Incubators' holistic/open process provides several opportunities for feedback between 'the people' and the 'local authority' to voice opinions on a design. A key aspect of the TRANSPLUS model is its ability to clearly communicate complex relationships without marginalising the significance of interactions between these relationships. 


\section{(o) NANTES}

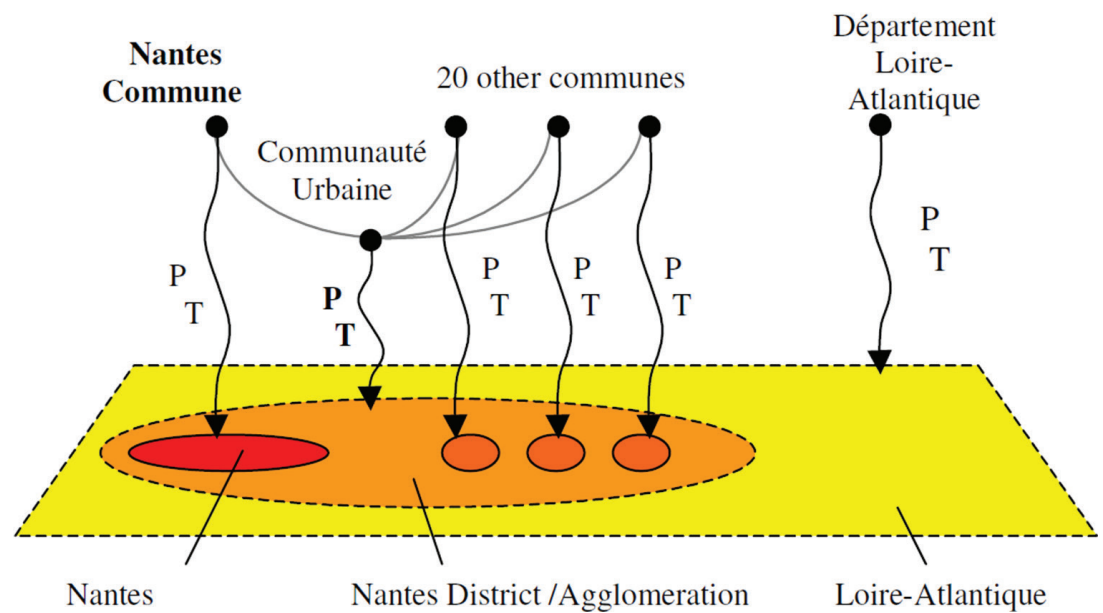

Figure 2: The areas, actors and instruments of Nantes as modelled by TRANSPLUS. $\mathrm{P}=$ planning policy instrument; $\mathrm{T}=$ transport policy instrument.

Source: TRANSPLUS D4

\section{IDENTIFYING AND PLACING ACTORS AND INSTRUMENTS AT POLLARDS HILL}

In this section, the modelling and principles of the TRANSPLUS framework will be applied to the Incubators case study, Pollards Hill. This is specifically to document the interplay between the actor and instruments present in this case study. To comply with the London Plan, local authorities have drafted local development frameworks that high priority areas for the provision of town centres, housing, open spaces and so on. In the case of the London Borough of Merton, this document is in the form of the Merton Sites and Policies Plan. Within the plan, only open space policies CS13 and DM01 affect Pollards Hill (covering Donnelly Green). CS13 essentially relates to the 'protection and enhancement of open space and creating opportunities for culture, sport, recreation and play facilities' [15] with loss of this land only being for the accommodation of educational facilities; DM01 echoes SP13 but goes on to stress the need for any necessary developments on this land to enhance the character of the space [15].

The ward of Pollards Hill is subject to a Neighbourhood Improvement Plan that concerns residential properties at the case study. Moat owns $36 \%$ of the homes at this site. The plan aims to engage more children and young people in positive activities, improve the physical appearance of the Pollards Hill estate, improve the reputation of the estate and reduce crime.

Focusing on the project of the Pollards Hill Estate, constraints effecting grassroots activities can be noted in the Moat Homes Tenancy Agreements (MHTA), Moat Homes Residents' handbook (MRH) and the Moat Tenants' handbook (MTH) [16]. Constraints included here include the proper maintenance of gardens in nonspecific terms [16]. These same rules appear to apply to the subdivision of sites, although local by-laws may become apparent following site surveys (of signs prohibiting or allowing activities) and/or the conducting of interviews with the local residents. Additionally, social interactions-human-spatial rules and bylaws remain detached from the recognised policies at Pollards Hill. 
The rules concerning human-spatial relationships are applicable to the courtyards and are independent of bylaws and mutual agreements. In other words, it is not expected that the courtyards will influence these ground rules as these rules are generic-having been honed from studies carried out in various urban spaces [17,18]. These rules will determine the placement of smaller objects throughout the site such as allotment plots and benches by individual users of the software platform.

Based on observations made during site visits, varying bylaws are apparent across the case study's courtyards. Also apparent, is that mutual understandings between the residents play a role in uses deemed acceptable throughout the courtyard spaces. These bylaws and rules are only applicable to the courtyard geographies towards the north of the site. Mutual understandings can potentially be as many as every other resident. As such this is a process of structuration [19], where even the smallest action contributes to the formation or alteration of a social system. The opted solution to this is cybernetics [12] taking the form of rules that govern the parameters of acceptable submissions.

Included in Fig. 3 is a blue tile that represents the underlying influence of human-spatial relationships that were formulated into rules during the Incubators literature review [12]. In the development of the software platform, these rules are applicable to all of the courtyard spaces owing to their generic nature. It is important to acknowledge that the purpose of these rules is to ensure good design practice in situations where the designing will be carried out by non-designers; as such automation will not only ensure design parameters are assured, but will also reduce unnecessary variety apparent in the process.

This sentiment exists between the identified bylaws and mutual understandings at Pollards Hill, where these feed into the functionality of the courtyard; however, there is always the possibility that rules could be waived based on architectural modifications such as the introduction of hardscaping that could introduce further rules such as 'no skateboarding, rollerblading, and scooters', where the point here is that the courtyards can potentially influence the by-laws and mutual understandings present leading to a dialogue between these

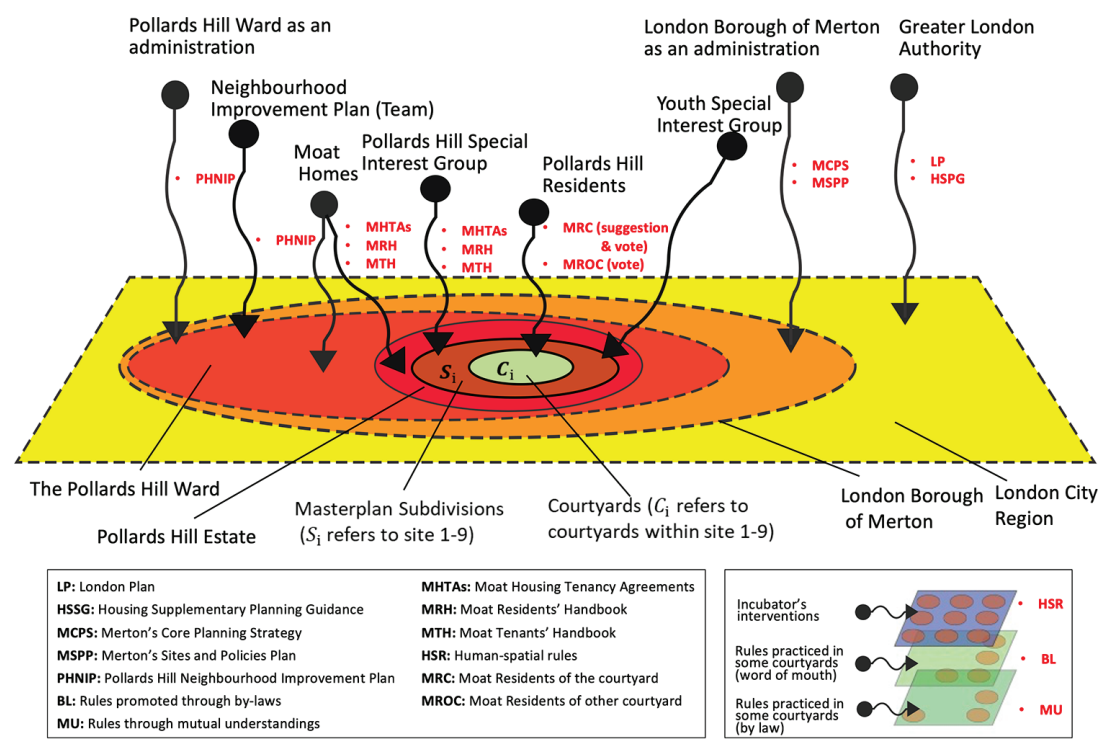

Figure 3: A TRANSPLUS framework representation of Pollards Hill. 
rules and physical composition of the courtyards themselves (this possible conundrum has yet to be modelled).

Social network analysis of the relationships between the networks reveals, using degrees of centrality measures - the number links that connect to a node [20], that the plots at Pollards Hill are directly influenced by external MRH, MTH, and MHTA instruments. These Moat published documents are in turn influenced by inputs from consulted Moat tenants as well as government policy instruments provided by Merton that are influenced by the Greater London Authority. The policy instruments are almost exclusively generated outside of the courtyards, although community groups associated with the plots and courtyards potentially play greater roles in designated use of the space.

The plots at the site are also influenced by the informal codes presented in the form of bylaws and mutual agreements; however, within the context of Incubators, it is forecasted that human-spatial relationships are, or will play, a more significant role in determining the nature of activities that can occur within the individual courtyards.

Modelling relationships using a modified TRANSPLUS framework helps with predicting possible scenarios in relation to the implementation of incubators platforms by targeting particular platform users.

For example, with this information, it is possible to enable only users that have a direct interest to the urban design area to intervene in the design process; in contrast, the data can also inform on who gets the right to vote, or suggest who may be external to the process. Fig. 4 shows four possible scenarios:

- (I) For a given courtyard, only residents are able to create proposals and vote on them;

- (II) For a given courtyard, only residents are able to create proposals, while both they and others from other courtyards may vote on the proposals;

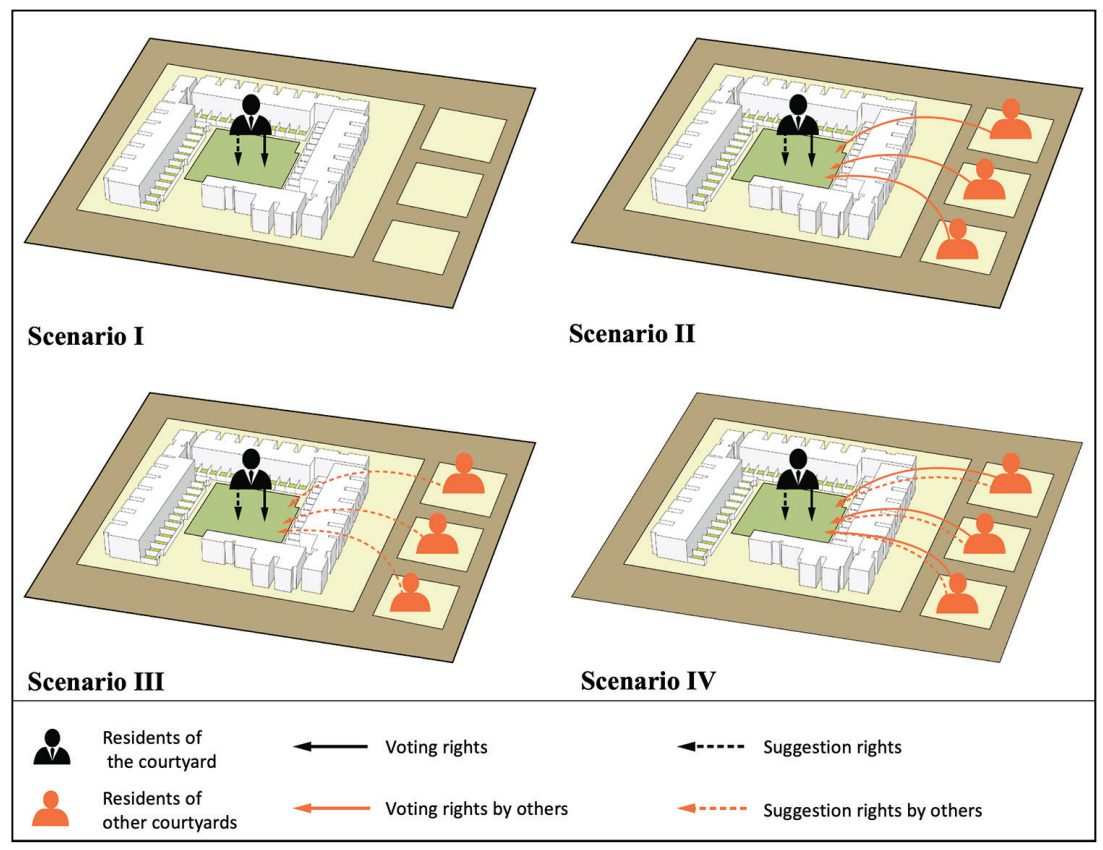

Figure 4: Pollards Hill's projected Incubators platform scenarios. 


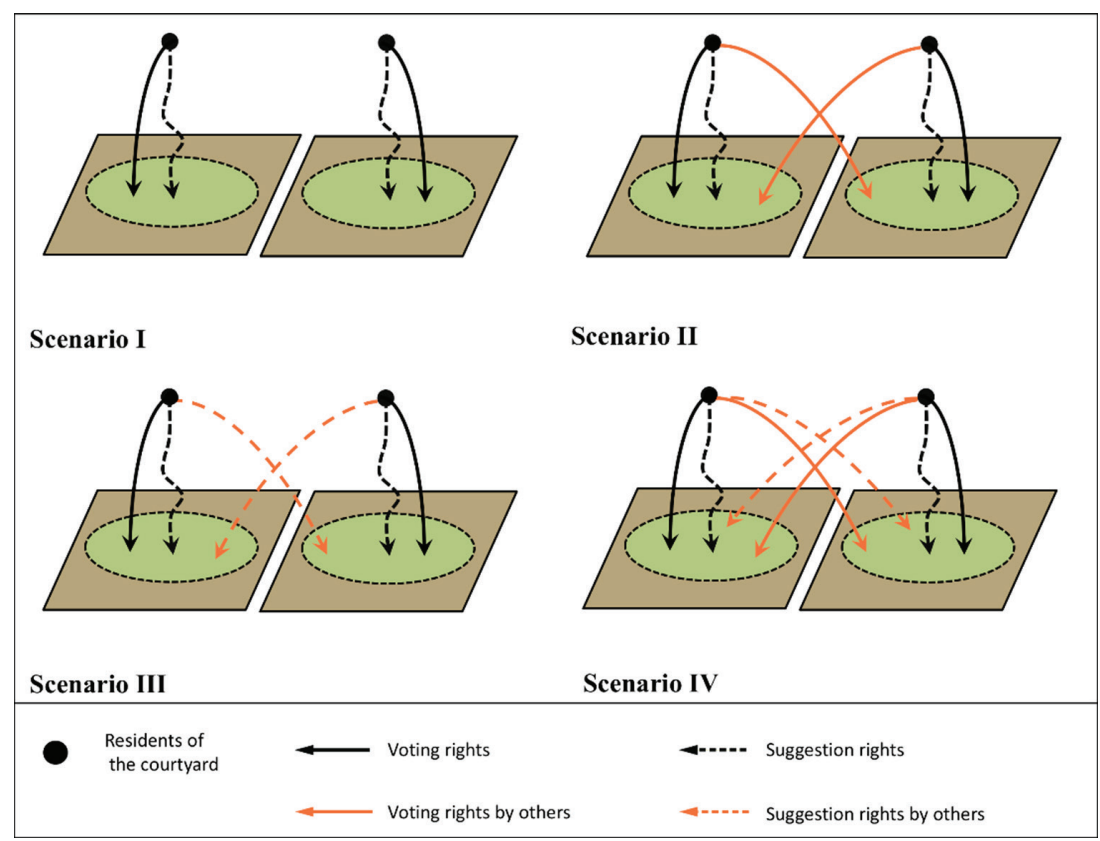

Figure 5: Pollards Hill scenarios modelled using the adapted TRANSPLUS framework.

- (III) For a given courtyard, both residents from that courtyard and from other courtyards are able to create proposals, but only residents of that particular courtyard are able to vote on the final proposal;

- (IV) Residents of all courtyards are able to create and vote for proposals for any courtyard.

In the Pollards Hill case, scenario II was initially proposed as the preferred model, since it was felt that all residents of all courtyards had a stake in the outcome (Fig. 5). That said, by the time the platform was actually implemented (August 2017), the project ran with a single generic courtyard as its model, for everyone to make proposals for and everyone to comment on (more like scenario IV).

\section{DISCUSSION}

By discussing layers of actors, areas and instruments within the context of TRANSPLUS, this paper highlights concerns surrounding community access to planning consultation processes at case studies like Pollards Hill and who has the right to decide the future of its morphological growth. It raises questions as to whether the process of courtyard-to-courtyard consultation should be opened up to become co-creative processes with everybody having the right to vote on an idea, facilitated by the Incubators platform.

Smart technologies exploit data collected from a variety of accessible consumer apps, providing insightful data that optimises both the validity and efficiency of mundane processes. The broad demographic captured through smart media is desirable in planning consultation exercises, where conventional methods often fail. Failure to engage a broad demographic in participatory design harms not only the sustainability of a project but also the 
sustainability of the affected community. However, conventional modelling tools fail to acknowledge the influence of policy instruments and marginalised actors involved in the gradual growth and development of areas such as the courtyards at Pollards Hill.

This paper principally tested the effectiveness of an adapted application of the TRANSPLUS method as a tool for visualising relationships between areas, actors and instruments. Applying TRANSPLUS to Pollards Hill recognises that seemly small aspects-by-laws, mutual understandings, human-spatial rules, informal interactions-play a collectively significant role in how an urban space is perceived and subsequently used, affecting its sustainability. The adaptation of the methodology allows the developers of Incubators and similar projects to identify the user groups that are least affected by conventional consultation techniques and that are most likely to use a smart-technology-based tool like Incubators to express their vision for their local urban space(s). This adaptation also highlights the necessity for participatory planning approaches to consider real-time planning approaches, offered by platforms like Incubators, when dealing with communities who aren't involved in consultation processes [21].

The social sustainability of a community largely depends on how facilities are perceived and used. Getting this right in at an initial phase is difficult for most planners because they either adopt a skewed approach by targeting specific individuals or focus groups within a community and/or struggle to rally community interest in their regeneration of their local spaces/facilities. Part of this problem is down to where they pitch their ideas, where the application of the TRANSPLUS approach, which relies on SNA and content analysis, can aid professionals in identifying disengaged community members and determining an appropriate approach towards engaging a broader demographic in the creation of well used spaces.

\section{ACKNOWLEDGEMENTS}

The research leading to these results has received funding from Joint Programming Initiative Urban Europe, under Grant 414896. The Partners of the Incubators of Public Spaces Consortium are Politecnico di Torino (coordinator), Innovation Service Network GmbH, Katholieke Universiteit Leuven, Neurovation GmbH, University College London and Turin Municipality. The UCL part of the project was funded by the UK Economic and Social Research Council (ESRC), Grant ref: ES/M008495/1. The authors are grateful to all who have supported or contributed to this project.

\section{REFERENCES}

[1] Book: Haughton, G. \& Hunter, C., Sustainable Cities, Routledge, p. 218, 1996. https:// doi.org/10.2307/144514

[2] Journal article: Yeung, P., Passmore, A. \& Packer, T., Examining citizenship participation in young Australian adults: a structural equation analysis. Journal of Youth Studies, 15(1), pp. 73-98, 2012. https://doi.org/10.1080/13676261.2011.623689

[3] Journal article: Arnstein, S.R., A ladder of citizen participation. Journal of the American Institute of Planners, 35(4), pp. 216-224, 1969. https://doi. org/10.1080/01944366908977225

[4] Journal article: Alawadi, K. \& Dooling, S., Challenges and opportunities for participatory planning approaches within Dubai's urban context. Journal of Urbanism: International Research on Placemaking and Urban Sustainability, 9(3), pp. 276-301, 2016. https://doi.org/10.1080/17549175.2015.1045924

[5] Journal article: Marcus, B.L., Public participation in planning: an intellectual history. Australian Geographer, 36(3), pp. 283-299, 2005. https://doi.org/10.10 80/00049180500325694 
[6] Online: Mayor of London. Smart London. https://london.gov.uk/what-we-do/businessand-economy/science-and-technology/smart-london (accessed 29 September 2017).

[7] Conference: Ding, C., Wald, M. \& Wills, G., A survey of open accesibility data. Proceedings of 11th Web for All Conference, 37, pp. 1-4, 2014. https://doi.org/10.11 45/2596695.2596708

[8] Book: Brabham, D.C., Crowdsourcing, John Wiley \& Sons, Inc., 2013.

[9] Report: Marshall, S., TRANSPLUS: D4-Barriers, Solutions and Transferability, University College London: London, 2003.

[10] Journal article: Nyström, A.G., Leminen, S., Westerlund, M. \& Kortelainen, M., Actor roles and role patterns influencing innovation in living labs. Industrial Marketing Management, 43(3), pp. 483-95, 2014. https://doi.org/10.1016/j.indmarman.2013.12.016

[11] Online: Moat. Closer to home: Local update for Moat residents living in Pollards Hill. https://moat.co.uk/uploadedFiles/About_Moat/Press_Office/Resident_publications/10. $\% 20$ Closer\%20to\%20Home\%20-\%20Pollards\%20Hill\%20-\%20Spring\%202014.pdf (accessed 29 September 2017).

[12] Report: Magnani, G., Timmerman, R. \& Karadimitriou, N., Work Package 2: Deliverable 2.1-Literature Review, University College London: London, 2016.

[13] Journal article: Iveson, K., Cities within the city: do-it-yourself urbanism and the right to the city. International Journal of Urban and Regional Research, 37(3), pp. 941-956, 2013. https://doi.org/10.1111/1468-2427.12053

[14] Online: Office of National Statistics. UK Census Data: Pollards Hill. http://ukcensusdata.com/pollards-hill-e05000467\#sthash.rfjD03MS.dpbs (accessed 29 September 2017).

[15] Online: London Borough of Merton. Core Planning Strategy. https://www2.merton. gov.uk/environment/planning/planningpolicy/localplan/core_strategy.htm (accessed 29 September 2017).

[16] Online: Moat: Residents' handbook. https://moat.co.uk/uploadedFiles/Residents/Residents\%20renting\%20landing\%20page\%20Residents\%20handbook.pdf (accessed 29 September 2017).

[17] Book: Gehl, J. \& Svarre, B., How to Study Public Life. Island Press, 2009.

[18] Book: Whyte, W., The Social Life of Small Urban Spaces, Project for Public Spaces, 1980.

[19] Book: Stones, R., Structuration Theory. New York, NY: Palgrave Macmillan, 2005.

[20] Book: Carrington, P.J., John, S. \& Stanley, W. (eds), Models and Methods in Social Network Analysis, Vol. 28. Cambridge University Press, 2005.

[21] Journal article: Marshall, S., Hudson-Smith, A., Farndon, D. \& Kourniotis, A., Digital participation - taking 'Planning' into the third dimension. Town and Country Planning, 88(1), pp. 11-14, 2019. 